\title{
Management of the subclavian steal syndrome
}

\author{
P. J. MOLLOY AND E. WYN JONES \\ From the Regional Cardio-thoracic Surgical Unit, Broadgreen Hospital, and the Regional Cardiac \\ Centre, Sefton General Hospital, Liverpool
}

Since the subclavian steal syndrome was first described by Contorni in 1960, numerous articles have appeared indicating that it is commoner than was formerly realized. Reivich, Holling, Roberts, and Toole (1961) drew the attention of the English literature with an account of two patients who had features of cerebral ischaemia, and its striking title was coined in an editorial (1961) in the New England Journal of Medicine. The present paper describes five such patients and seeks to outline the steps in management and to discuss problems arising therefrom.

The underlying pathology in nearly all the recorded cases is atherosclerotic occlusive vascular disease, although arteritis, dissecting aneurysm, or syphilis may give rise to the condition. Diabetes may be associated (Siekert and Millikan, 1955) with basilar artery insufficiency states.

In patients with the subclavian steal syndrome occlusion of the subclavian artery (or innominate artery) proximal to the vertebral artery on that side gives rise to a pressure differential between the two vertebral arteries. Blood from the contralateral vertebral artery is siphoned over at the basilar junction and flows retrograde into the subclavian artery on the occluded side, thereby depriving the basilar artery of some of its supply (Fig. 1). If the carotids are stenosed the brain stem circulation may be imperilled by a lack of flow through the anastomotic channels at the circle of

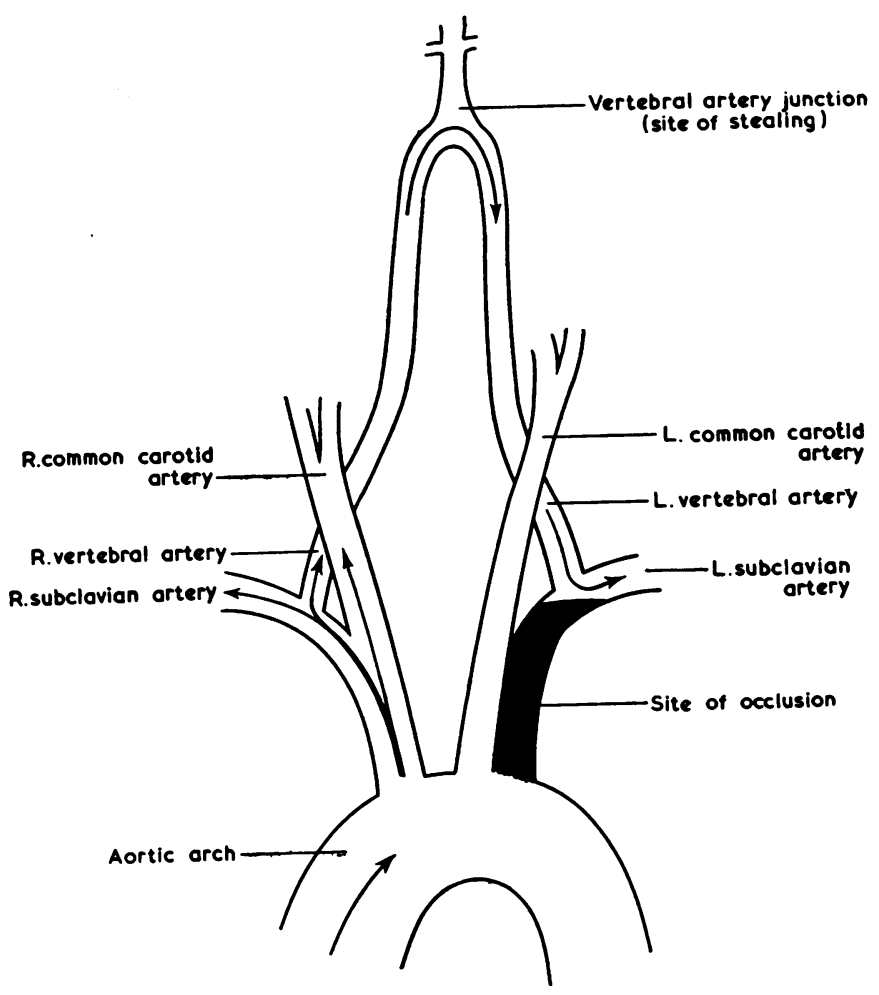

FIG. 1. The arrows show the direction of blood flow when the left subclavian artery origin is occluded, indicating the site of subclavian stealing at the basilar artery junction. 
Willis, as is evidenced in the third patient in this group. Rotation of the head may aggravate symptoms due to occlusion of the prograde flowing vertebral artery.

\section{CASE REPORTS}

CASE 1 J.H. aged 61, a right-handed ship's engineer. awoke in Hamburg in August 1964 with dysarthria and a left arm paresis. He had a controlled atrial fibrillation of unknown aetiology of 11 years' duration. Physical examination showed signs of generalized atheroma, with no other abnormality in the cardiovascular, respiratory, or alimentary systems. The left hand was colder than the right, in which arm the blood pressure was $165 / 105$ $\mathrm{mm}$. Hg. The left arm pressure was $100 / ? \mathrm{~mm}$. $\mathrm{Hg}$. There were no bruits in the root of the neck. Aortography, using percutaneous femoral artery puncture by the Seldinger technique, showed obstruction to the left subclavian artery with reversed flow down the left vertebral artery (Fig. 2).

On 17 February 1965 operation was performed through a left fourth rib bed posterolateral incision. The aorta was taped, and the left subclavian artery and its branches were dissected free and held in nonocclusive tapes. The subclavian artery was opened after partly occluding the aorta and the subclavian artery origin, and endarterectomy was performed. The plaque extended into the aorta, and on opening the aortic clamp no prograde flow ensued. A $4 \mathrm{~mm}$. disc of the occluding plug was excised, but it was thought that the flow was inadequate. A $\frac{5}{8}$ in. $(16 \mathrm{~mm}$.) Teflon graft was sutured end-to-side from the aorta to the distal end of the subclavian incision. Flow on releasing the aortic clamp was satisfactory. The patient's convalescence was uneventful and he was discharged from

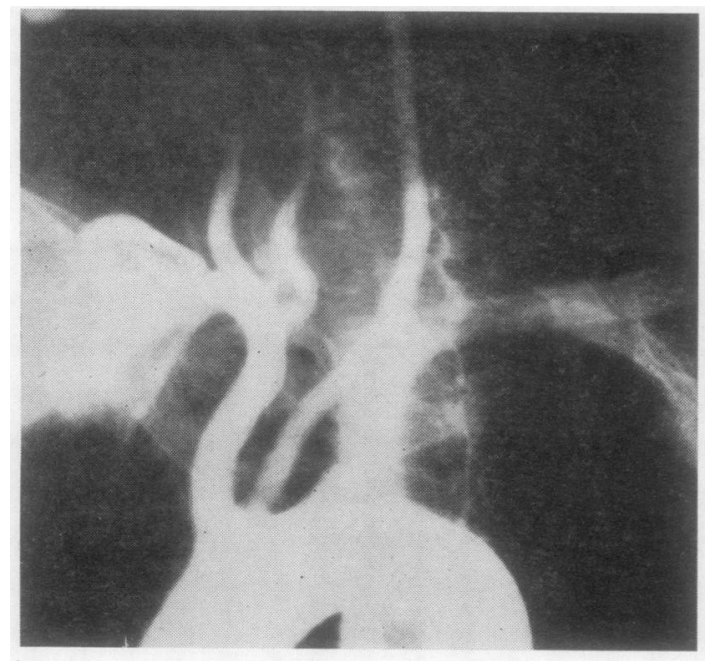

(a) hospital with equal pressures in the two arms $(160 / 100 \stackrel{\overrightarrow{\vec{S}}}{\vec{s}}$ $\mathrm{mm} . \mathrm{Hg}$ ). He survived a myocardial infarct six weeks $\overline{0}$ later and has been maintained throughout on anticoagulants. He has had no further episodes of cerebral ischaemia in 12 months of survey.

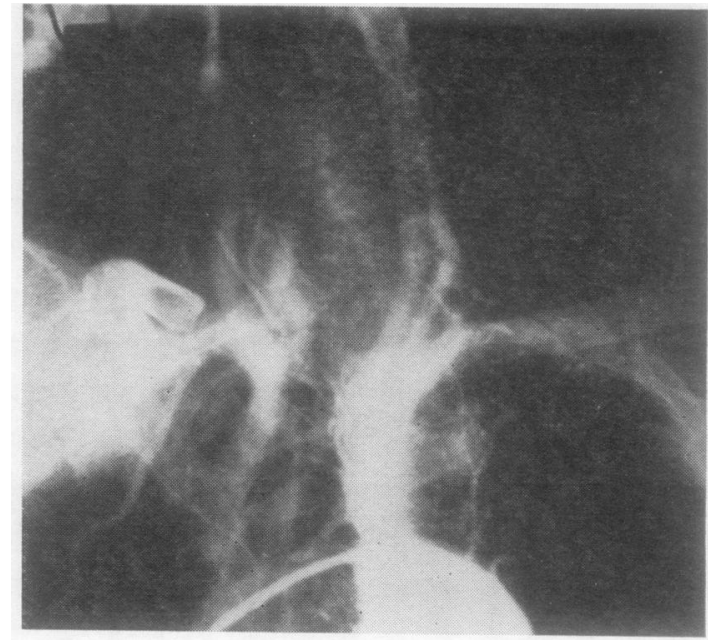

(b)

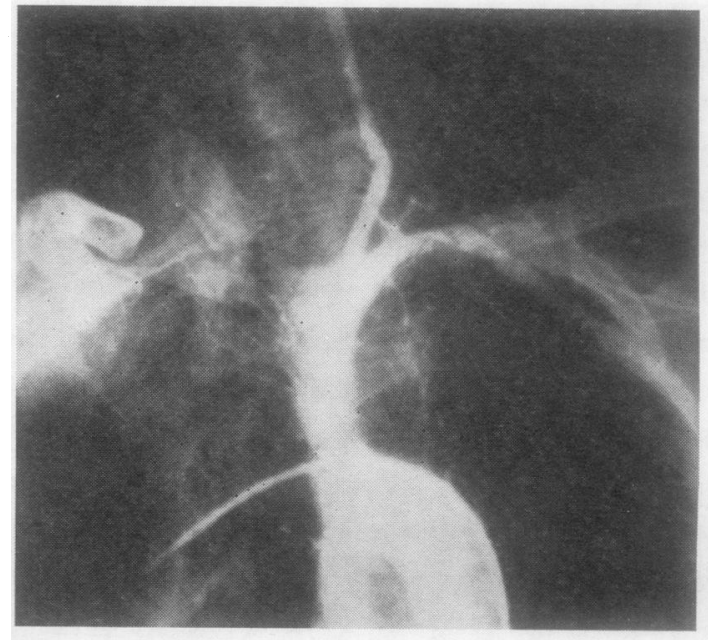

(c)

FIG. 2. Case 1. Retrograde aortogram, left oblique view. (a) Shows normal innominate and left carotid arteries with the right vertebral artery filled. There is only a short stump of the left subclavian artery visible; (b) 1.5 sec. after (a) shows left vertebral artery filling retrogradely; (c) $0.5 \mathrm{sec}$. after (b) shows left vertebral artery still opacified and filling the left subclavian artery. 
Comment This patient showed no hypertension and no arm claudication, nor were any other symptoms provoked by arm exercise.

CASE 2 M.P. aged 61, a right-handed school-teacher, had an 11 months' history of dizziness, loss of speech, weakness in the left arm, and occasional syncopal attacks. Turning the head to the left or using the left arm induced dizziness. An electrocardiogram showed changes of left ventricular hypertrophy. She had been maintained on anticoagulants and hypotensive drugs for some time before admission.

An arch aortogram (femoral route, Seldinger technique) showed occlusion of the left subclavian artery from its origin to the origin of the vertebral artery, which was filled retrograde from the right vertebral artery. The other arch vessels showed changes of atherosclerosis. In February 1965 the left subclavian artery was explored through a left fourth rib bed incision. Because of the widespread atherosclerosis an aorto-subclavian graft using $\frac{5}{8}$ in. $(16 \mathrm{~mm}$.) Teflon was performed by side-to-side anastomosis. There was good flow on releasing the clamp, and convalescence was uneventful. Adequate anticoagulation control was difficult to obtain due to an idiosyncrasy until warfarin was substituted for phenindione, but she was relieved of symptoms and has maintained equal pressure in each $\operatorname{arm}(190 / 100 \mathrm{~mm}$. $\mathrm{Hg})$.

Comment This patient showed limb claudication and symptoms related to exercise and posture in a known hypertensive.

CASE 3 T.A. aged 58, a social worker, also righthanded, had had intermittent dysphagia for two weeks and visual disturbances for two years. She had had a known systemic hypertension for five years (B.P. 180/ $100 \mathrm{~mm}$. Hg). She presented when she had a drop attack at the school of the previous patient's husband, who made the correct diagnosis! There was no limb claudication, but the syncopal attack had been preceded by loss of power in the left arm. She showed pulse differences (R. 100/0; L. 185/105 mm. Hg) with no neurological signs in the limb. There were, however, right and left carotid bruits, a to-and-fro murmur over the right subclavian origin, and bruits over each renal artery. An electrocardiogram showed minor changes of left ventricular hypertrophy. An arch aortogram (percutaneous femoral artery, Seldinger technique) showed the left subclavian artery to be patent, the left vertebral artery narrowed at its origin, the left carotid artery occluded, and the right subclavian artery blocked to the origin of the right vertebral artery, in which blood flow was retrograde. The right internal carotid artery was also occluded. This patient's cerebral circulation depended on a stenosed left vertebral artery (Fig. 3). After the aortogram she had three dizzy attacks interpreted as a portent of worsening cerebral ischaemia and therefore had an emergency operation.

The innominate artery was approached through a low transverse neck incision, and, although a little cramped by the medial end of the clavicle, exposure was adequate for proximal control. The innominatesubclavian artery junction was opened, and an occluding plaque of atheroma was removed up to the origin of the vertebral artery. Since the right common carotid artery was patent above the innominate artery bifurcation, the carotid bifurcation was exposed through a higher crease incision and a right internal carotid endarterectomy was performed. There was surprisingly good back-bleeding down the internal carotid artery. After closure of the incisions there was good prograde flow in both vertebral and carotid arteries.

It was elected to continue with anticoagulants. She was well for two days with equal pressures of 200/110 $\mathrm{mm}$. $\mathrm{Hg}$ in each arm ; 72 hours post-operatively she had an epileptic seizure and became comatose and hemiplegic. Her blood pressure was noted to be $270 /$ $180 \mathrm{~mm}$. Hg. She failed to regain consciousness and died 76 hours after operation. Post-mortem examination showed the sites of endarterectomy to be patent, and the cause of death was massive subarachnoid and intracerebral haemorrhage occasioned by the hypertension and possibly aggravated by the anticoagulants.

Comment This patient showed no limb features other than pulse difference, but was a known hypertensive. More adequate control of the acute hypertension while she was on anticoagulants might have averted the loss of this patient.

CASE 4 A.M. aged 48, a clerical worker, had had vertiginous attacks for two and a half years and diplopia for seven to eight months. There was no limb claudication nor neurological disturbance. There was a pulse discrepancy (blood pressure R. 80/60, L. $130 / 70 \mathrm{~mm}$. $\mathrm{Hg}$ ) and a loud systolic bruit on the right at the root of the neck. An arch aortogram (percutaneous femoral, Seldinger technique) showed marked atheromatous narrowing of the innominate artery with early poor filling of the right subclavian artery. The later films showed retrograde flow through the right vertebral artery and later refilling of the right subclavian artery.

The aortic arch was explored through a median sternotomy which gave excellent exposure. A right innominate and subclavian endarterectomy was performed with restoration of prograde flow. During the procedure the right carotid circulation was supported by a femoro-carotid bypass. Hypothermia was not used. Convalescence was uneventful and at discharge the patient was symptom free with equal pressures in each arm $(140 / 90 \mathrm{~mm}$. $\mathrm{Hg})$.

Comment This patient had progressive symptoms of vertebral ischaemia and was not hypertensive. Although there was some prograde flow in the right subclavian artery, the pressure differential was sufficient to permit retrograde filling of the right vertebral artery and subclavian stealing. 


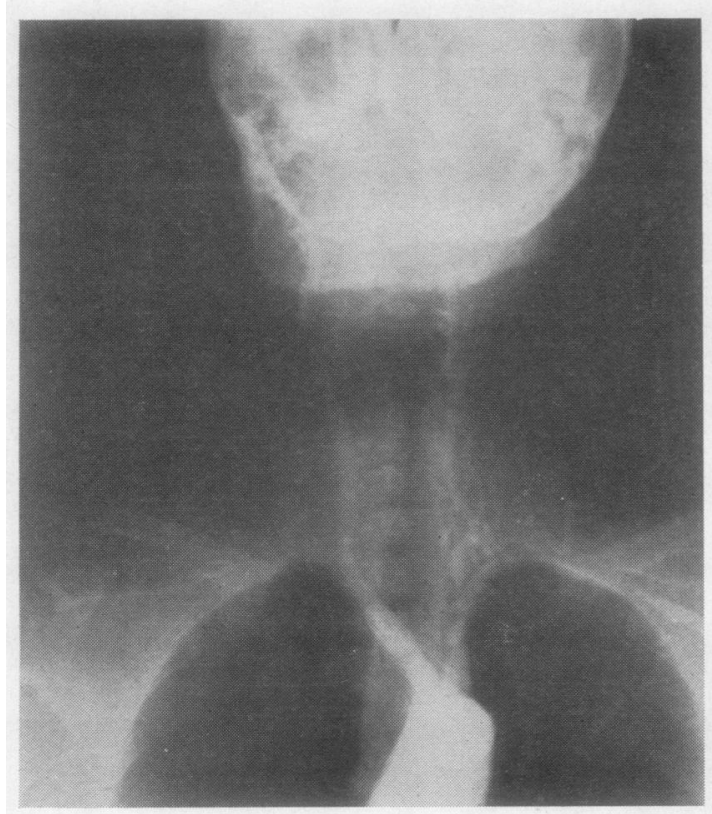

(a)

FIG. 3. Case 3. Retrograde aortogram, A.P. view. (a) Shows innominate and left subclavian arteries filling with absent internal carotid arteries on each side. The only prograde cerebral flow visible is via the atheromatous left vertebral artery; (b) $1 \mathrm{sec}$. after (a) shows the right vertebral artery starting to fill retrogradely; (c) $1 \mathrm{sec}$. after (b) shows the right vertebral artery well filled and opacifying the right subclavian artery; $(d)$ diagram to explain $(b)$. The arrows indicate the direction of the blood flow.

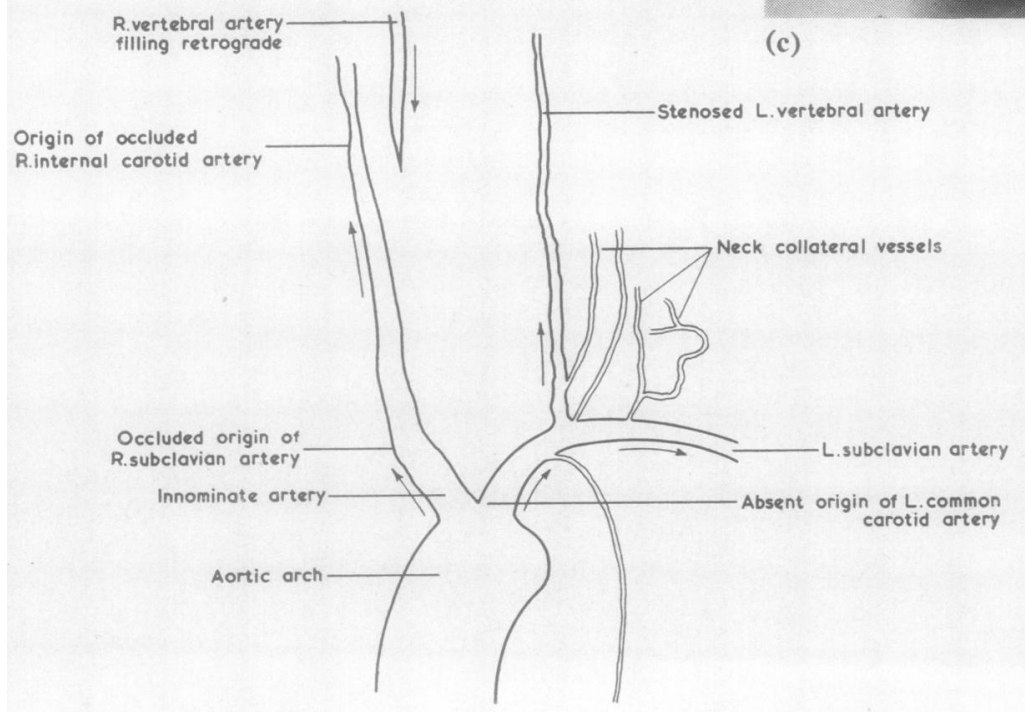

(b)

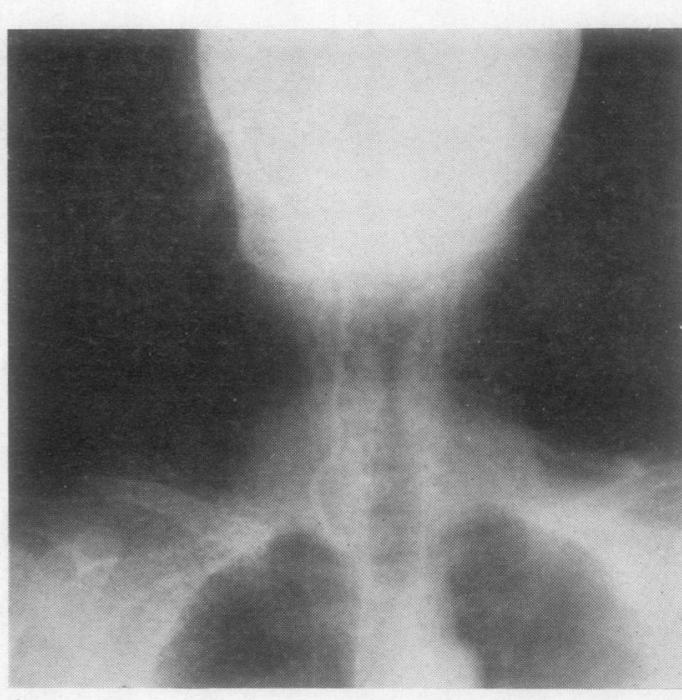

ב

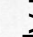

극

N

N

N

음

0

D 


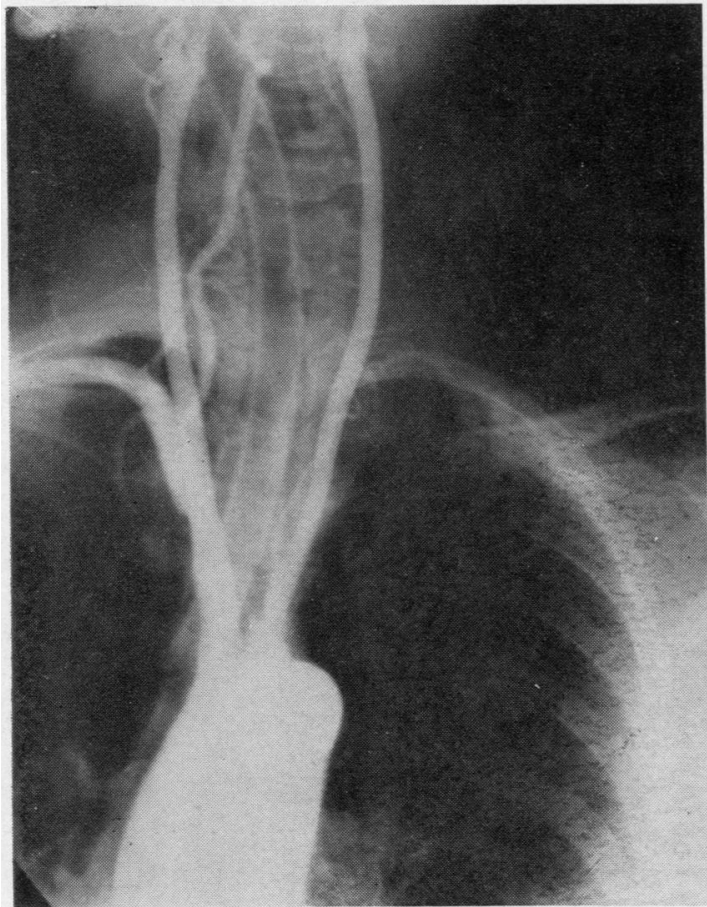

(a)

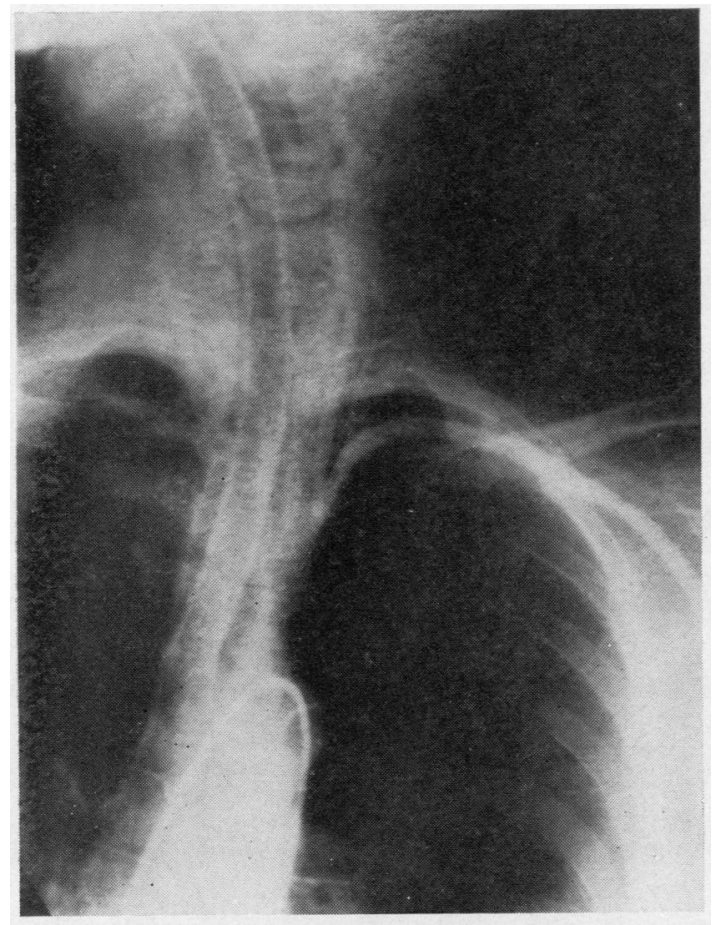

(c)

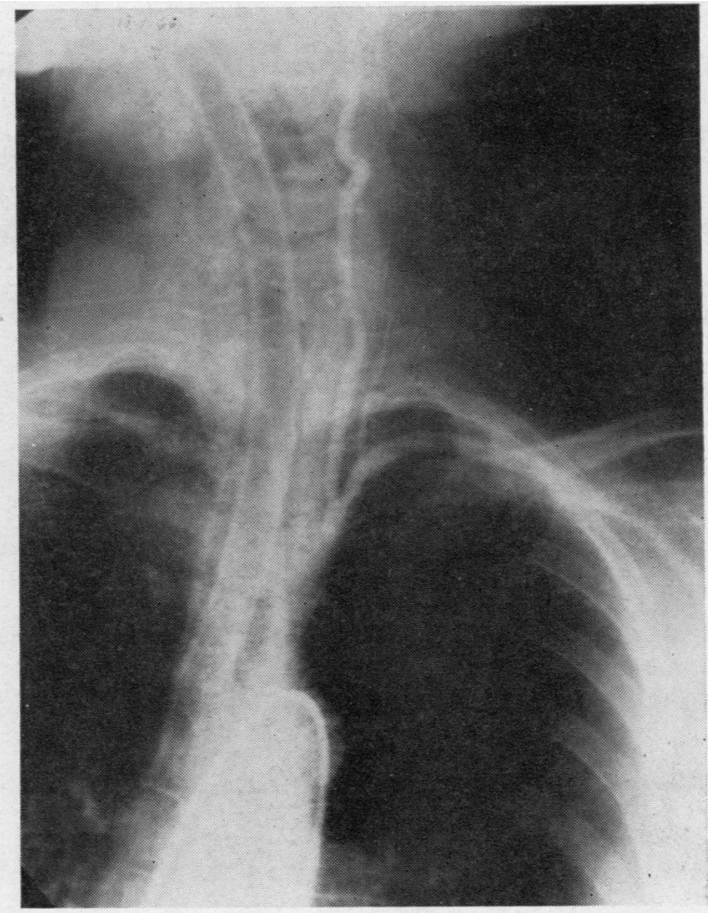

(b)

FIG. 4. Case 5. Retrograde aortogram, semi-oblique view. (a) Shows the innominate, right subclavian and vertebral, and left carotid arteries well filled, with absent left subclavian artery; (b) 2.5 sec. after (a) shows the left vertebral artery filling retrogradely with early opacification of the left subclavian artery; (c) $0.5 \mathrm{sec}$. after (b) shows the left subclavian artery strongly opacified, indicating a signifcant degree of 'steal.' Note the small stump of subclavian artery proximal to the origin of the vertebral artery on the left.

CASE 5 R. McD. aged 56, an electrician, noticed numbness of the left arm, paraesthesia, and pain brought on by working overhead with his left arm. He had had vertigo off and on for four years. He had coldness of the fingers in the left hand and occasional numbness. Turning his head to the left brought on dizziness and aggravated his symptoms. Examination showed a cold left hand with diminished pulse (blood pressure: right $150 / 70 \mathrm{~mm}$. $\mathrm{Hg}$, left $100 /$ ? $\mathrm{mm}$. $\mathrm{Hg}$ ). The left subclavian artery could not be felt. There were no bruits. The remainder of the examination was uninformative. An aortogram (right transfemoral route) showed an absent left subclavian artery with late retrograde flow down the left vertebral artery (Fig. 4). Operation was performed on 18 January 1966. On exposing the left subclavian artery through a left third rib bed incision it was clear that the vessel was blocked right to its origin. Accordingly, an aorto-subclavian bypass graft 
was performed. There was good prograde flow on releasing the clamps. Convalescence was uneventful. He has been relieved of his symptoms, and blood pressure is equal in each arm at $160 / 100 \mathrm{~mm}$. $\mathrm{Hg}$.

Comment This patient was not hypertensive but had limb claudication and a postural exacerbation of symptoms.

\section{DISCUSSION}

The problems that arise in the management of this condition are those of diagnosis, treatment, and post-operative care.

DIAGNOSIS Symptoms are non-specific unless arm claudication or postural changes worsen them. The neurological features may suggest many nonvascular and vascular cerebral lesions, such as disseminated sclerosis, pseudobulbar palsy, neurovascular syphilis, cervical vertebral spondylitis, scalenus anticus syndrome, diabetic neuropathy, basilar artery sclerosis, or cerebral vascular sclerosis. Many of these patients are in an age group in which degenerative disease is common, and unless the examiner is aware of the subclavian steal syndrome as a cause of these symptoms the diagnosis will be overlooked.

Hypertension is not necessarily a feature and was not present in our cases 1,4 , and 5 . In a study of 54 patients with vertebrobasilar insufficiency, Bradshaw and McQuaid (1963) noted that 37 were hypertensive. The patients may have other features of occlusive vascular disease as manifest by leg claudication, myocardial ischaemic symptoms, or temporal arteritis.

The critical points of diagnosis (apart from awareness of the condition) are differentiation of the pulses in the two arms and adequate radiography. Bruits should be sought, and Peart and Rob (1960) have emphasized the value of listening over the sites of division of vessels for evidence of partial occlusion. A totally occluded vessel will of course, give rise to no flow murmur. Both radial and brachial pulses on each side should be compared by palpation, as there may be only 20 to $30 \mathrm{~mm}$. $\mathbf{H g}$ difference. The blood pressure should be taken in each arm. The finding of a pulse discrepancy is the most useful clinical sign in the diagnosis of subclavian obstruction, and, if associated with symptoms of cerebral ischaemia, the diagnosis of a subclavian steal syndrome should be entertained. Williams, Scott, and Takaro (1963) have shown that the pulse in the subclavian artery on the affected side may be obliterated by pressing on the origin of the ver- tebral artery in the neck on that side. They also emphasize the relation of postural changes of the arm and neck to the onset of symptoms.

Accurate anatomical localization of the site of obstruction and demonstration of the reversed vertebral flow is only possible by performing aortography. It is not enough to visualize the carotid artery on the affected side, as all the arch vessels must be seen. Sufficient late films must be taken to show filling of the vertebral vessels and the direction of their flow. After the initial descriptions of this condition, radiologists confirmed the patho-physiological circulation and established it as a radiological entity (Simon, Rabinov, and Horenstein, 1962 ; Philp, Samuel, and Duncan, 1963 ; Ashby, Karras, and Cannon, 1963 ; Steinberg and Halpern, 1963 ; Fischer and Mattey, 1963). Great care, especially in the elderly (Williams and Wilson, 1962), must be taken, and retrograde percutaneous femoral artery puncture is to be preferred to the brachial route, as postangiogram thrombosis of the subclavian artery on the non-affected side may provoke bilateral vertebral, and consequent basilar, artery thrombosis as well as upper limb ischaemia. Using a Seldinger technique, gentle advancement of the guide wire will permit the catheter to advance to the aortic arch.

Subclavian artery thrombosis is more serious than femoral artery thrombosis, which must also be recognized as a possible complication as the limb circulation will be endangered. As in case 3, the cerebral circulation may be so imperilled that the hypertonic contrast medium may provoke ischaemic symptoms. If these patients are being investigated, facilities for rapid surgical treatment either of the lesion itself or of complications of the investigation must be available.

TREATMENT Recognition of the presence of the condition is not sufficient indication for treatment. The patients should have progressive symptoms sufficient to alter their normal way of life and should not be frail or elderly, since operative management inflicts a major hazard, especially in the left-sided lesions. In patients whose symptoms do not warrant operative treatment, or whose condition does not permit it, there is a place for prolonged anticoagulant treatment with dicoumarol derivatives, bearing in mind the risk of cerebral or other haemorrhage, especially if the patient is hypertensive. Bradshaw and McQuaid (1963) noted a poor prognosis in their series of cases with vertebro-basilar insufficiency especially in those patients denied anticoagulants. These 
authors found an $8 \%$ mortality in 101 patients on anticoagulants, but a $50 \%$ mortality in a smaller series of similar patients not so treated.

Operative procedures should aim at disobliterating the obstructed segment or bypassing it. There is little place for simple vertebral artery ligation, except perhaps in the frail or elderly with severe symptoms which progress in spite of anticoagulant therapy. There is a high risk of basilar artery thrombosis, which is always fatal (French and Haines, 1950).

On the left side the subclavian artery is approached via the left fourth rib bed. This gives good access to the aortic arch, the subclavian artery origin, and its first branches. The aorta should be taped above and below the subclavian artery to allow control of haemorrhage. If the origin of the vessel is patent, as determined by aortogram, palpation, and aspiration of blood, it can be clamped at its origin, and an endarterectomy can be performed through a small (1 in.; $25 \mathrm{~mm}$.) incision.

If the lumen appears narrowed a vein patch or woven material patch can be inserted to widen it. Care must be taken that the proximal limit of the endarterectomy does not extend into the aortic lumen, as not only may this act as the site of a dissecting aneurysm but the atheromatous aortic wall may disintegrate and result in an uncontrollable haemorrhage.

If the subclavian artery is totally obliterated at its origin and the aortic wall as seen on the aortogram is atheromatous, it is better to bypass the obstruction with a graft from the aorta distal to the subclavian artery to the region of the subclavian branches. A localized endarterectomy may be necessary distally in the subclavian artery to give an adequate lumen proximal to the vertebral artery, but too much intimal removal need not be attempted.

On the right side, if the innominate artery is blocked the arch of the aorta should be approached by a median sternotomy using the full length of the sternum. There is less risk of opening the pleura, healing is as good and postoperative discomfort a good deal less than a $\mathrm{T}$ shaped incision in the sternum. If access over the subclavian artery is desired, the upper end of the incision can be extended across the neck 1 in. $(25 \mathrm{~mm}$.) above the clavicle with or without the detachment of the sternal and clavicular heads of sternomastoid. There is little place for excising the medial end of the clavicle. The aorta and innominate artery can be dissected free and controlled. The innominate vein should be dissected free along its full visible length and gently retracted to expose the innominate artery bifurcation: good exposure is obtained by this method. As on the left, endarterectomy or bypass graft can then be performed to restore the lumen.

If the segment of the subclavian artery between the innominate artery bifurcation and the vertebral artery origin alone is blocked, the obstruction may be approached by a simple neck incision from the left of the suprasternal notch to the junction of the outer and middle thirds of the clavicle. The cervical pleura should be carefully retracted to avoid inducing a pneumothorax after elevating the heads of the sternomastoid. After taping the innominate, subclavian, and carotid arteries endarterectomy can be performed. It must be noted that side clamping of the innominate-carotid junction is necessary to allow carotid artery flow. If this is not practicable, facilities for femoro-carotid bypass or hypothermia should be available. The use of hypothermia adds considerably to the risks of the operation and should not be employed routinely, as it has its own hazards (Cooper and Ross, 1960). If necessary, a carotid endarterectomy can be performed at the same time, as in case 3 .

POST-OPERATIVE CARE It is the authors' practice to give $50 \mathrm{mg}$. heparin $(5,000$ units) once vascular incisions are closed and any leak controlled, and this is repeated after six hours in the belief that heparin anticoagulation minimizes platelet deposition on the vascular suture line in the early post-operative thrombotic phase. If there is no good evidence of widespread vascular disease, as in case 5, anticoagulants are stopped forthwith. If atheroma is widespread, long-term anticoagulation should be continued for the rest of the patient's life, with exact and adequate control. If laboratory facilities are not adequate it is probably safer to rely on the flow through the restored vessels. Irvine, Luck, Sutton, and Walpita (1963) advise against anticoagulants early because of the danger of bleeding from suture lines, but note that complications are seldom serious in patients on long-term therapy.

If the patients are hypertensive great care must be exercised in controlling this feature, especially in the anticoagulated patient. In case 3 , failure to do this resulted in loss of the patient. Chlorpromazine or ganglion-blocking agents in small doses intravenously give the best control during the acute phase.

Drainage is not used in the neck incision routinely, but, if necessary, suction drainage for 24 hours only is employed. 


\section{SUMMARY}

Five patients with the subclavian steal syndrome are described.

Problems arising in diagnosis, management, and post-operative care are discussed with particular reference to the need for controlling postoperative hypertension in an anticoagulated patient.

The authors wish to thank Dr. E. J. Epstein, who. referred case 1, and Mr. Raymond Helsby, who referred cases 4 and 5. The central Photographic Unit of the University of Liverpool and Mr. D. Allen made the photographic copies of the aortograms.

\section{REFERENCES}

Ashby, R. N., Karras, B. G., and Cannon, A. H. (1963). Clinical and roentgenographic aspects of the subclavian steal syndrome. Amer. J. Roentgenoi., 90, 535.

Bradshaw, P., and McQuaid, P. (1953). The syndrome of vertebrobasilar insufficiency. Quart. J. Med., 32, 279.

Contorni, L. (1960). Il circelo colletrale vertebro-vertebrale nella obliterazione dell'arteria subclavia alla sua origine. Minerva chir., 15, 268.
Cooper, K., and Ross, D. N. (1960). Hypothermia. In Surgical Practice. Cassell, London.

Editorial (1961). A new vascular syndrome-'the subclavian steal'. New Engl. J. Med., 26j, 912.

Fischer, M. J., and Mattey, W. E. (1963). The subclavian steal syndrome. Amer. J. Roentgenol., 90, 532.

French, L. A., and Haines, G. L. (1950). Unilateral vertebral artery ligation. Rep ort of a case ending fatally with thrombosis of the basilar artery. J. Neurosurg., 7, 156.

Irvine, W. T., Luck, R. J., Sutton, D., and Walpita, P. R. (1963). Intrathoracic occlusion of great vessels causing cerebrovascular insufficiency. Lancet, 1, 1177.

Peart, W. S., and Rob, C. (1960). Arterial auscultation. Ibid., 2, 219.

Philp, T., Samuel, E., and Duncan, J. G. (1963). Reversed vertebral artery blood flow in subclavian artery obstruction. Clin. Radiol., 14, 310 .

Reivich, M., Holling, H. E., Roberts, B., and Toole, J. F. (1961). Reversal of blood flow through the vertebral artery and its Reversal of blood flow through the vertebral artery and
effect on cerebral circulation. New Engl. J. Med., 265, 878 .

Siekert, R. G., and Millikan, C. H. (1955). Some clinical aspects of thrombosis of the basilar artery. Proc. Mavo Clin., 30, 93.

Simon, M., Rabinov, K., and Horenstein, S. (1962). Proximal subclavian artery occlusion and reversed blood flow to the arm. Clin. Radiol., 13, 201.

Steinberg, I and Halpern, M. (196i)), Roentgen manifestations of the subclavian steal syndrome. Amer. J. Roentgenol., 90, 528.

Williams, C. L., Scott, S. M., and Takaro, T. (1963). Subclavian steal. Circulation, $28,14$.

Williams, D., and Wilson, T. G. (1962). The diagnosis of the major and minor syndromes of basilar insufficiency. Brain, 85, 741 . 